\title{
Complete Resolution of a Refractory Pyogenic Granuloma Following Topical Salt Treatment
}

\author{
Nouf Faihan Bin Rubaian ${ }^{1,2}$ \\ 'Dermatology Department, King Fahad \\ Hospital of the university, Imam \\ Abdulrahman bin Faisal University, \\ Dammam, Saudi Arabia; ${ }^{2}$ Department of \\ Dermatology, College of Medicine, Imam \\ Abdulrahman bin Faisal University, \\ Dammam, Saudi Arabia
}

\begin{abstract}
Salt is an inexpensive, widely available substance that has few treatment side effects, apart from a mild stinging sensation that resolves after topical application. Salt has proven to be an effective treatment for pyogenic granulomas in children and leads to rapid lesion resolution without recurrence. We report a case of a young girl in whom salt is used as a treatment for a partially excised pyogenic granuloma, with complete lesion resolution.
\end{abstract}

Keywords: pyogenic granuloma, salt

\section{Introduction}

The term "pyogenic granuloma" (PG) is a misnomer for a mass that is neither infectious nor granulomatous. PG is a benign vascular growth that presents as a friable red papule or polyp on the gingiva, finger, lip, face, or tongue of a patient. Such lesions bleed following a minor trauma. PGs are seen more commonly in children and young adults than in older patients; males are affected to a slightly greater extent. In affected pregnant women, lesions often involve the gingiva; hence, the term granuloma gravidarum was coined. ${ }^{1}$

Treatment modalities include surgical excision, electrocautery, cryotherapy, pulsed dye laser treatment, and intralesional sclerotherapy using monoethanolamine oleate. Topical therapy with timolol $0.5 \%$ ophthalmic gel and imiquimod $5 \%$ cream is also used.

Topical salt application has recently been described as a treatment for PG and showed a successful outcome in our patient who had a residual lesion after surgical excision. ${ }^{1-3}$

\section{Case Report}

An 11-year-old Saudi girl presented to the emergency room with hemorrhage from a swelling in the right half of the lower lip that had appeared 1 month earlier. Digital pressure and the act of eating caused the lesion to bleed. Avoidance of eating had led to weight loss in the patient.

The lesion was an erythematous friable papule, measuring $1 \mathrm{~cm}$ in diameter, on the right half of the lower lip (Figure 1). The patient had no history of chronic illness or medication use prior to the development of the swelling. The clinical appearance of the lesion and the history of bleeding associated with trauma suggested a diagnosis of PG.

Treatment at our clinic included shave excision of the lesion using a blade, followed by electrocautery and silver nitrate application to control bleeding. When bleeding did not stop, two silk sutures were used to close the wound.
Correspondence: Nouf Faihan Bin Rubaian

P.O. Box 1982, Dammam, 3|44I, Saudi

Arabia

Tel +966 I3-3330030

Fax +966 I3-3330108

Email cm.dir@iau.edu.sa;

nbinrubaian@iau.edu.sa 


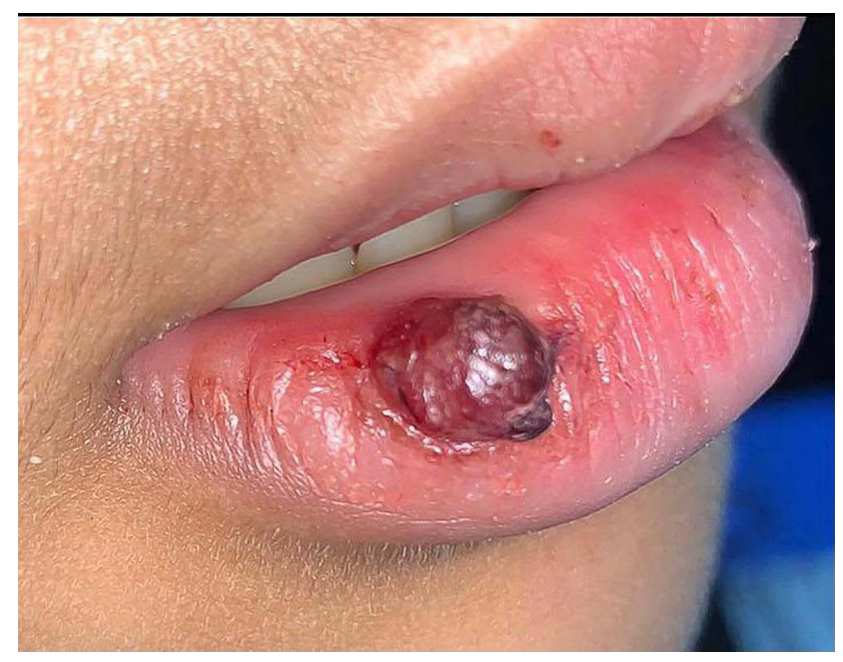

Figure I Lesion at presentation as erythematous friable papule, measuring $\mathrm{I} \mathrm{cm}$ in diameter, on the right half of the lower lip.

Following suture removal 5 days later, further treatment was required to address incomplete lesion excision (Figure 2). Two cycles of cryotherapy were performed but were not tolerated by the patient. Thus, the patient's mother was advised to administer a treatment at home that involved 1) applying table salt to the lesion, 2) coating the perilesional skin with Vaseline, and 3) covering the wound with a gauze. One week later, the lesion was markedly reduced in size and consisted of a small papule (Figure 3). To reduce irritation of the lesion caused by direct salt application, home treatment was continued with cotton buds soaked in salt water that were applied to the lesion one to two times per day. After 2 weeks of salt treatment, the patient's lesion had completely resolved (Figure 4).

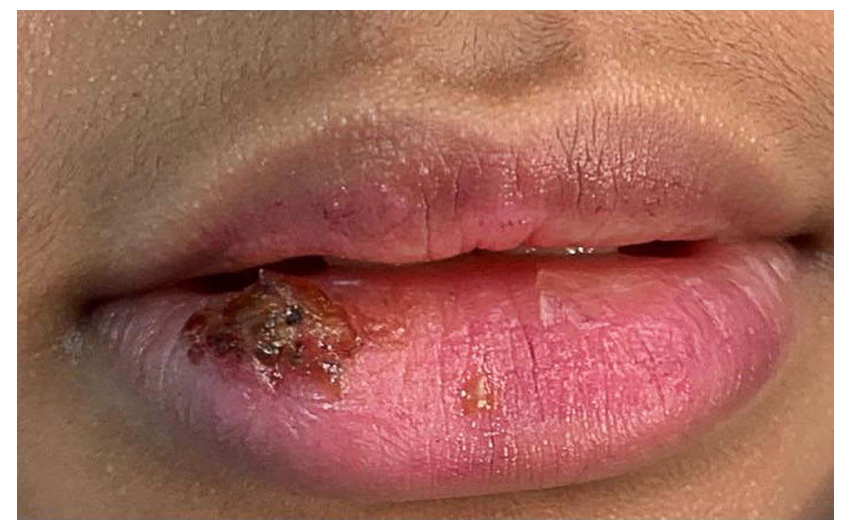

Figure 25 days after suture removal of the partially excised lesion when a two cycles of cryotherapy was done.

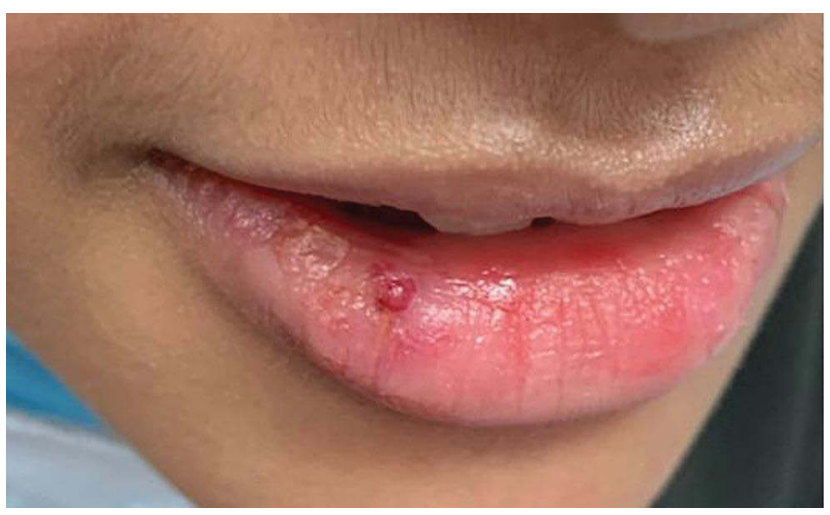

Figure 3 After one week of topical salt use, the lesion was markedly reduced in size and became a small papule.

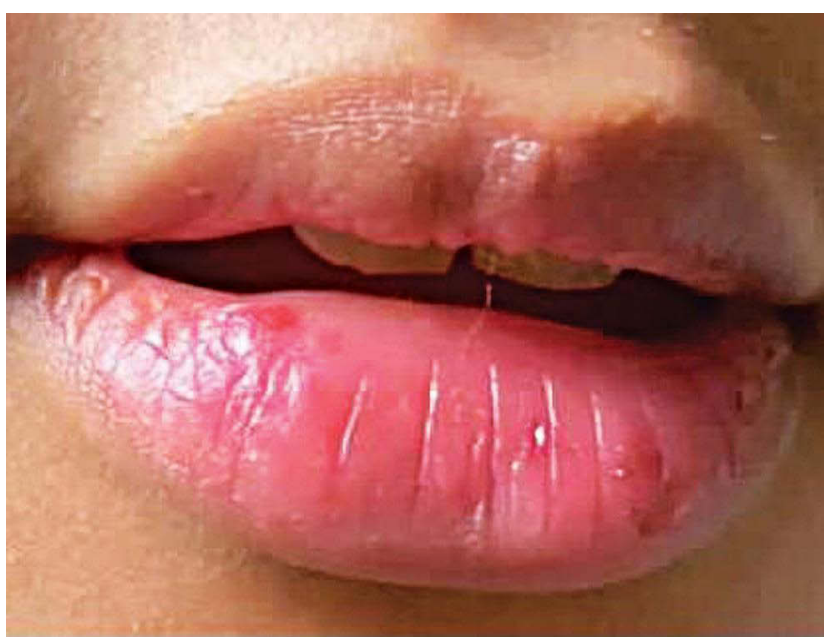

Figure 4 Complete resolution of the lesion after two weeks of topical salt.

\section{Discussion}

PB is a benign vascular neoplasm that can occur as a solitary lesion or multiple lesions. Lesion development is associated with systemic medications such as retinoids, indinavir, and BRAF and EGFR inhibitors. ${ }^{1,2}$

These markedly friable masses ulcerate and bleed easily, and usually require surgical resolution. Satellite lesions can appear near the primary PG and on the accompanying area of "port-wine stain". ${ }^{1}$ The macroscopic lateral tissue margins of PG are often defined by epithelial collarettes. ${ }^{1}$ Histologically, PG appears as a circumscribed proliferation of small capillaries arranged in a lobular pattern and surrounded by fibromyxoid stroma.

Treatment options for PG include surgical excision, shave excision, laser surgery, electrodesiccation, curettage, liquid nitrogen cryotherapy, sclerotherapy, topical silver nitrate application, and topical imiquimod application. Recurrence is common, due to inadequate removal or 
incomplete destruction of the lesions. Surgical excision with linear closure has the lowest recurrence rate and is considered the treatment of choice; however, scarring is a major disadvantage with this treatment method, especially at sites such as the face where cosmetic appearance is a concern for patients. Among the non-surgical treatment options, liquid nitrogen is used most often and has the lowest overall recurrence rate, followed by carbon dioxide laser ablation and flashlamp pulsed dye laser therapy. ${ }^{4,5}$

Salt has been recently described as a treatment for PG. Daruwalla et al reported a case in which a pinch of salt was applied to a lesion, soft white paraffin was applied at the lesion margins to prevent perilesional skin irritation, and the lesion was covered with a surgical adhesive tape. Resolution of the PG occurred within 7-14 days of treatment; no recurrence was observed at the 1 month followup. Our patient, whose lesion resolved completely within 2 weeks, had a similar outcome.

Daruwalla et al conducted a prospective open-label uncontrolled study in which 50 patients with PGs were treated with salt application. Complete lesion resolution with no residual scarring was observed in $100 \%$ of the patients, and $94 \%$ of the patients reported a decrease in bleeding from their lesions shortly after treatment (mean of 3.7 days after treatment), followed by a gradual reduction in lesion size until complete resolution. The mean time from treatment to complete resolution was 14.77 days. In the study by Daruwalla et al, nearly $33 \%$ of the participants (15 out of 50) were pediatric patients, whose satisfaction with treatment was assessed with a "smiley face" survey. All patients reported being "highly satisfied" $(91 \%)$ or "satisfied" $(9 \%)$ with salt treatment. A total of $95 \%$ of the patients stated that they would repeat salt therapy for any recurrences of PG, and the remaining 5\% of patients stated that they would prefer a treatment that provided faster results. ${ }^{4}$

Salt application to PG creates a hyperosmolar tissue environment that leads to lesion shrinkage due to a desiccant effect. ${ }^{2,3}$ Salt has also been used to treat umbilical granulomas with an efficacy comparable to that achieved with silver nitrate, copper sulfate, and surgery. Salt treatment resulted in a $100 \%$ initial cure rate with no recurrence after 1 month and only one recurrence reported by Daruwalla et al 11 months after prior resolution. Patients whose lesions did not respond well to salt treatment were misdiagnosed or achieved partial responses that were fully resolved when salt treatment was extended. $4,6,7$
Although PGs occur in patients of any age, they are more prevalent in children, adolescents, and pregnant women. The mean age of onset among children is 6.7 years. PGs account for $0.5 \%$ of all childhood skin nodules and most often arise on a patient's face. Salt application is an excellent treatment for PG, based on the sites of lesion development, young patient age, absence of scarring, and frequency of complete lesion resolution. ${ }^{4}$

Apart from a mild reversible stinging sensation during application, salt treatment has no complications; therefore, it can be used in children, in patients unwilling to undergo surgical excision, at sites where cauterization is difficult, or on large lesions that require reduction prior to excision. $^{2,3,6,7}$

\section{Conclusion}

Salt is an inexpensive, widely available substance with almost no treatment side effects, apart from a reversible mild stinging sensation. Salt treatment has been used to achieve rapid resolution of PGs, with a very few recurrences observed. It is a good choice to treat PG in children, elderly patients who cannot undergo even a minor procedure or on sites where a scar may be of a concern as the face.

\section{Ethical Statement}

The article does not contain the participation of any human being or animal. Informed Consent was taken since the study presents findings for a human patient.

\section{Informed Consent/Institutional Approval}

Informed consent was obtained from the patient and her mom for publication of the case details and accompanying images. This case report did not require institutional approval, as per the IRB guidelines of our hospital. The case study was conducted in a manner that observes the provisions of the Declaration of Helsinki.

\section{Acknowledgments}

Dr. Mohamad Al-Shahrani and Dr. Sarah Al-Breiki for reviewing the case report. Dr. Amaal Alghamdi for supervising the paper writing with suggested modification and helping in the final draft of the case report and helped in the reply to the reviewers' comment. Dr. Ghada Alyousif for suggesting to elaborate more in the section of case presentation. Dr. Amna Almulhim for technical help. 
Lama Alnaimi for English editing. Noof aldossary for her support in enhancing the clarity of the figures.

\section{Disclosure}

The author has no conflicts of interest for this work to disclose.

\section{References}

1. Daruwalla SB, Dhurat RS. A pinch of salt is all it takes! The novel use of table salt for the effective treatment of pyogenic granuloma. $J \mathrm{Am}$ Acad Dermatol. 2020;83(2):e107-e108. doi:10.1016/j.jaad.2019.12. 013

2. Haftu H, Bitew H, Gebrekidan A, Gebrearegay H. The outcome of salt treatment for umbilical granuloma: a systematic review. Patient Prefer Adherence. 2020;14:2085-2092. doi:10.2147/PPA.S283011
3. Haftu H, Gebremichael TG, Kebedom AG. Salt treatment for umbilical granuloma - an effective, cheap, and available alternative treatment option: case report. Pediatr Health Med Ther. 2020;11: 393-397. doi:10.2147/PHMT.S269114

4. Daruwalla SB, Ghate S, Dhurat R. Establishing the efficacy and safety of the novel use of common salt for the treatment of pyogenic granuloma. Clin Exp Dermatol. 2021. doi:10.1111/ ced. 14658

5. Lee J, Sinno H, Tahiri Y, Gilardino MS. Treatment options for cutaneous pyogenic granulomas: a review. J Plast Reconstr Aesthet Surg. 2011;64(9):1216-1220. doi:10.1016/j.bjps.2010. 12.021

6. George NM, Potlapati A. Salt for the treatment of pyogenic granuloma in a patient with pustular psoriasis of palms. Clin Exp Dermatol. 2021;46(2):407-409. doi:10.1111/ced.14368

7. Bolognia JL, Schaffer JV, Cerroni L. Pyogenic Granuloma, benign vascular neoplasm and reactive hyperplasia. In: Bolognia JL, Schaffer JV, Cerroni L, editors. Dermatology. Vol. 2, 4th ed. Elsevier Health Sciences; 2018:2026-2027.

\section{Publish your work in this journal}

The Open Access Emergency Medicine is an international, peerreviewed, open access journal publishing original research, reports, editorials, reviews and commentaries on all aspects of emergency medicine. The manuscript management system is completely online and includes a very quick and fair peer-review system, which is all easy to use. Visit http://www.dovepress.com/testimonials.php to read real quotes from published authors. 